\title{
The Effect of Afatinib Treatment in Non-small Cell Lung Cancer Cells
}

\author{
JOANNA PANCEWICZ-WOJTKIEWICZ ${ }^{1}$ and PAWEL LESZEK BERNATOWICZ ${ }^{2}$ \\ ${ }^{1}$ Department of Histology and Embryology, Medical University of Bialystok, Bialystok, Poland; \\ ${ }^{2}$ Department of Hematology, Medical University of Bialystok, Bialystok, Poland
}

\begin{abstract}
Background: Non-small cell lung cancer (NSCLC) is a very aggressive and histologically heterogeneous type of lung cancer. The main problem in the treatment of NSCLC is chemoresistance and metastasis. Compared to other malignant tumors, many molecular mechanisms are dysfunctional in NSCLC. Epidermal growth factor receptor (EGFR) is one of the most frequently mutated genes in NSCLC. Materials and Methods: We investigated the effect of afatinib the against A549 NSCLC cell line, because it is active against mutated EGFR. Moreover, we aimed to investigate the mRNA level of HSPA5 (one of the heat shock proteins that can contribute in the downregulation of the EGF-signaling pathway) before and after afatinib treatment. Results: Afatinib treatment induced apoptosis and decreased levels of HSPA5 MRNA in cancer cells. Conclusion: Advanced analysis, might be helpful in understanding the mechanisms of relations between tyrosine kinase inhibition of EGFR and HSPA5 in lung cancer cells.
\end{abstract}

Lung cancer is a histologically diverse type of tumor and is one of the most common causes of cancer mortality in the world (13 ). More than half of the lung cancer patients have non-small cell lung cancer (NSCLC). Available therapy mainly depends on pathomorphological classification (3). The evaluation of new drugs targeting altered pathways and genes could improve monitoring and further treatment of NSCLC patients, although resistance and metastasis are still major complications. Therefore, analysis of morphological changes in tumor cells, as well as corresponding alterations in mRNA levels, appears to be relevant in understanding the biology of this type of cancer. Here, we present the significance of the heat shock protein

Correspondence to: Dr. Joanna Pancewicz-Wojtkiewicz, Department of Histology and Embryology, Medical University of Bialystok, Waszyngtona 13, 15-269 Bialystok, Poland. Tel: +48 857485456, e-mail: jpancewicz@ymail.com

Key Words: HSPA5 (GRP78), afatinib, EGFR, non-small cell lung cancer.. family A (Hsp70) member 5-HSPA5 (the other common name is GRP78- glucose regulated protein of $78-\mathrm{kDa}$ ) in NSCLC. It is one of the stress proteins constitutively expressed in cells. It can be overexpressed in stressful conditions, such as hypoxia which is the typical microenvironment of solid tumors. Nevertheless, overexpression of HSPA5 could have opposite results on tumor cells and its effect in cancer seems to be tissue dependent. Conferring to available data, HSPA5 is known to induce apoptosis in cancer cells (4). However, according to other outcomes, overexpression of HSPA5 can be associated with malignant transformation of tumors, increased metastasis and overall poor prognosis in some types of cancer (5-8). Therefore, in the current study we aimed to investigate the mRNA levels of HSPA5 after treatment with the novel tyrosine kinase inhibitor - afatinib. It is well known, that EGFR is often deregulated in NSCLC, a fact that implicates further treatment $(9,10)$. Moreover, there are some evidences suggesting that HSPA5 contributes in the down-regulation of the EGF-signaling pathway (11).

Consequently, we measured HSPA5 mRNA expression in A549 cells before and after treatment with afatinib. Furthermore, we examined the effect of afatinib treatment by evaluating morphological changes in cells. Based on our results, we suggest that treatment of A549 cells with afatinib induced apoptosis, and a progressive decrease in the level of HSPA5 mRNA, during the time course treatment.

\section{Materials and Methods}

Cell culture. A549 cells were obtained from the American Type Culture Collection (ATCC). Cells were cultured in RPMI-1640 containing $10 \%$ fetal bovine serum (FBS) and antibiotics. The cells were incubated at $37^{\circ} \mathrm{C}$ in a humidified environment with $5 \% \mathrm{CO}_{2}$. The cell culture reagents were purchased from ATCC.

RNA extraction and quality control

Total RNA was isolated from A549 cells using the mirVana miRNA isolation kit (Thermo Fisher Scientific, Waltham, MA, USA) according to the manufacturer's protocol. The $100-\mu 1$ resulting RNA extracts were stored at $-80^{\circ} \mathrm{C}$ prior to further processing. Quantity and quality of RNA were assessed using a UV/VIS spectrophotometer Nano Drop 2000c (Thermo Fisher Scientific, Waltham, MA, USA). The level of integrity required for 


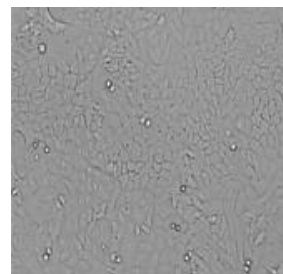

DMSO

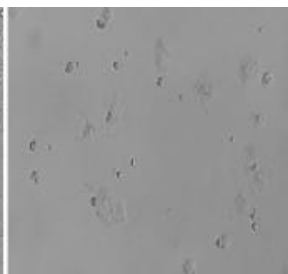

$24 \mathrm{~h}$

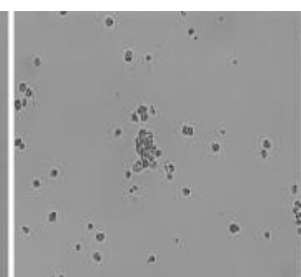

$48 \mathrm{~h}$

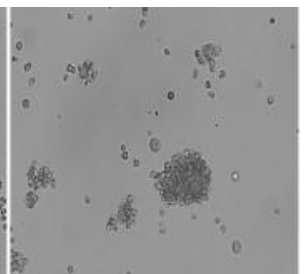

$72 \mathrm{~h}$

Figure 1. The results of morphological studies by converted microscopic examination (100x magnification). The A549 cells were treated with 10 $\mu M$ afatinib (tyrosine kinase inhibitor) during 24, 48 and $72 \mathrm{~h}$.

quantitation (RNA integrity number $>7$ ) was determined for the extracted total RNA using the Agilent RNA 6000 Nano Kit on a Bioanalyzer 2100 (Agilent Technologies, Santa Clara, CA, USA). A total of 500 ng RNA was reverse transcribed into cDNA in a reaction with High Capacity RNA-to-cDNA Master Mix (Applied Biosystems; Thermo Fisher Scientific, Waltham, MA, USA) according to the manufacturer's protocol.

Quantitative polymerase chain reaction $(q P C R)$. mRNA expression levels of HSPA5 were evaluated in the A549 cell line using comparative qPCR. The TaqMan probes (Hs00607129_gH) and the TaqMan Assay Kit (all from Applied Biosystems; Thermo Fisher Scientific, Waltham, MA, USA) were used to perform PCR. The expression of the above-mentioned genes [by the change-in-cyclingthreshold $\triangle \mathrm{Cq}$ method] (12) were calculated and normalized to ribosomal 18SRNA gene expression (Hs99999901_s1 18SRNA). The following cycling conditions were used: $50^{\circ} \mathrm{C}$ for $2 \mathrm{~min} ; 95^{\circ} \mathrm{C}$ for $10 \mathrm{~min} ; 40$ cycles of $95^{\circ} \mathrm{C}$ for $15 \mathrm{sec}$ and $60^{\circ} \mathrm{C}$ for $60 \mathrm{sec}$. Each sample was analyzed in triplicate. All reactions were performed using the ABI PRISM ${ }^{\circledR}$ 7900HT Sequence Detection system (Thermo Fisher Scientific, Waltham, MA, USA)

\section{Results}

Afatinib treatment caused A549 cell death. A549 cells were grown in 6-well plates and treated with afatinib. Treatment of cells was conducted for 24,48 and $72 \mathrm{~h}$. The concentration of afatinib used for the treatment was $10 \mu \mathrm{M}$. Morphological alterations of cells were noticed after $24 \mathrm{~h}$ of treatment. At that time, most of the cells detached from the plate, changed volume and structure, and their processes shrank. After 48 and $72 \mathrm{~h}$ of afatinib treatment, cells started to form clusters, which seemed to be greater in size after $72 \mathrm{~h}$ of treatment. The untreated cells $(1 \%$ DMSO only) formed a monolayer attached to the bottom of the plate, with no obvious morphological abnormalities (Figure 1). The index of apoptosis was determined by counting cells before and after treatment with afatinib. Around 500 cells in each well were counted, and based on morphological changes, we determined the number and percentage of apoptotic cells. Results showed that afatinib treatment increased the percentage of apoptotic cells (Figure 2).
HSPA5 expression in A549 cells. We examined the effect of afatinib in the expression of HSPA5 mRNA after treatment of A549 cells with afatinib. During the test with afatinib we collected cells, isolated RNA and check the mRNA expression level of HSPA5. Our baseline was A549 cells treated with DMSO only. Therefore, after $24 \mathrm{~h}$ of afatinib treatment, a significant decrease of HSPA5 mRNA level was observed (Figure 3). The experimental condition was the same as used for morphological microscopic observations.

\section{Discussion}

NSCLC is a histologically heterogeneous type of tumor (Figure 4) involving many dysfunctional molecular pathways including the EGFR pathway. In this study, we aimed to present the effect of treatment with a drug targeting altered EGFR in NSCLC. Hence, we selected afatinib which is a tyrosine kinase inhibitor active against mutated EGFR including T790M. Furthermore, we tried to find interactions between heat shock HSPA5 and the designated treatment. HSPA5 is known as glucose regulated protein of $78-\mathrm{kDa}$ (GRP 78) and is a member of the HSP family which is recognized to be overexpressed by ER stress (4). Consequently, we measured mRNA level of HSPA5 in the A549 cell line before and after afatinib treatment. Furthermore, we examined morphological changes in cells. Our studies conducted by microscopic examination (Leica DM 2000 LED light microscope (Leica Microsystems GmbH, Wetzlar, Germany) focused on general characterization of changes in the morphology of A549 cell line. We were able to observe changes in size (cell shrinkage), reduction in cell attachment and the formation of clusters. The results of microscopic examination indicated that afatinib treatment caused apoptotic modifications in cells. Moreover, our conclusion is consistent with latest research, that afatinib strongly induces mechanisms of apoptosis (13-15). Next, we examined the levels of HSPA5 mRNA before and after treatment, since accessible information conferring the role of HSPA5 in NSCLC, particularly in programmed cell death, is still contradictory. According to some current results, NSCLC cells treated with anti-HSPA5 antibodies showed reduced cell proliferation, colony formation, and enhanced 


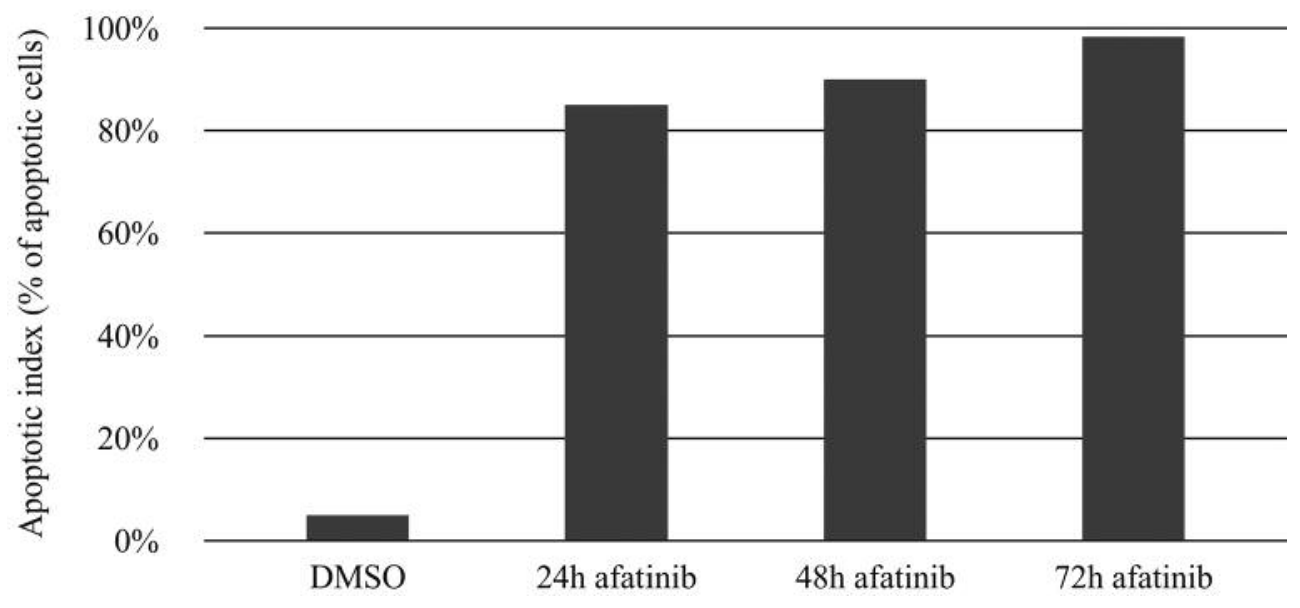

Figure 2. Percentages of A549 cell death via apoptosis after treatment with tyrosine kinase inhibitor (afatinib). The percentages of cell death via apoptosis increased significantly in a time-dependent manner.

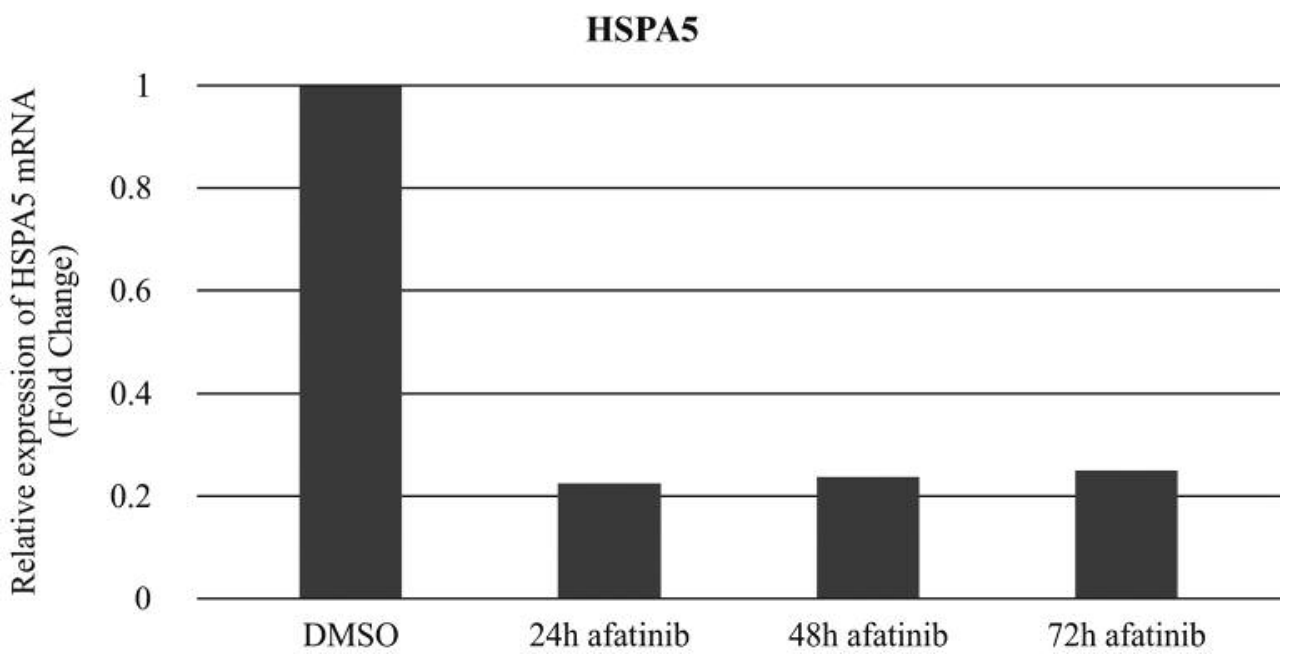

Figure 3. Relative expression of mRNA HSPA5 level in time course treatment with afatinib.

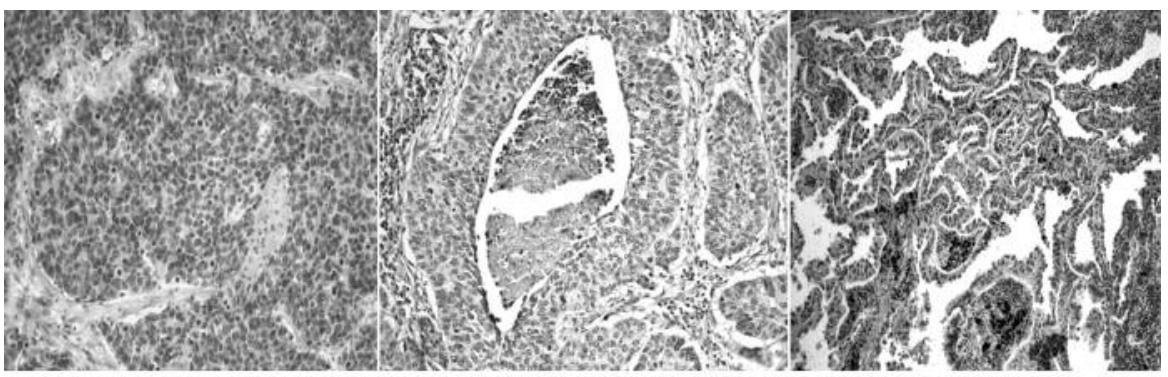
A) SCC
B) LCC
C) Adenocarcinoma

Figure 4. Representable picture of histological diversity of non-small cell lung cancer (NSCLC): SCC-Squamous Cell Carcinoma, LCC-Large Cell Carcinoma. 
apoptosis (16). Moreover, the experiments conducted by other researchers revealed that HSPA5 up-regulation in A549 cells followed by cisplatin treatment leads to increased apoptosis. Also, a newest study performed in cervical cancer cells and verified in animal model showed that increasing HSPA5 level caused stronger proliferation of tumor cells as well as influenced resistance to drug targeting cervical cancer. In accordance to results, it has been postulated by the authors, that high expression of HSPA5 (GRP78) is tumor stimulating in this type of cancer (17). In our experiment, the mRNA level of HSPA5 became lower during afatinib treatment. A549 cells changed morphology after $24 \mathrm{~h}$ of afatinib treatment and after $72 \mathrm{~h}$ cells were completely detached and formed clusters. Moreover, results of counting the cells indicated advanced apoptosis. Therefore, we think afatinib had apoptotic effect on A549 cell. Our data seems to be consistent with research conducted in breast cancer. The researchers proposed that plumbagin (anticancer drug) induces apoptosis in estrogenpositive breast cancer cells through HSPA5 (GRP78) inhibition (18). Nevertheless, in accordance to our studies afatinib by itself induces apoptosis and mRNA level of HSPA5 decreases. On the other hand, a study by Dadey Dy et al. showed that enhanced programmed cell death might be triggered by HSPA5 inhibition (16). In our results, it seems that apoptosis is caused by afatinib treatment and HSPA5 mRNA level reduction depends on morphological changes. However, our interpretations require further experiments, although it appears there is some connection between anticancer drugs apoptotic effect and HSPA5 in cancer cells (18).

\section{Conflicts of Interest}

The authors declare that there are no conflicts of interest regarding the publication of this study.

\section{Acknowledgements}

The present study was supported by the Budget for Science between the years 2013 and 2015, project no., IP2012 033872 (Iuventus Plus), to Joanna Pancewicz-Wojtkiewicz.

This study was conducted with the use of equipment purchased by Medical University of Bialystok as part of the OP DEP 20072013, Priority Axis I.3, contract No. POPW.01.03.00-20-022/09.

\section{References}

1 Aggarwal A, Lewison G, Idir S, Peters M, Aldige C, Boerckel W, Boyle P, Trimble EL, Roe P, Sethi T, Fox J and Sullivan R: The State of Lung Cancer Research: A Global Analysis. J Thorac Oncol 11: 1040-1050, 2016.

2 World Cancer Report IARC, WHO Press, Lyon, France, 2014.

3 Siegel RL, Miller KD and Jemal A: 2015 Cancer statistics. CA Cancer J Clin 65: 5-29, 2015.

4 Ahmad M, Hahn IF and Chatterjee S: GRP78 up-regulation leads to hypersensitization to cisplatin in A549 lung cancer cells. Anticancer Res 34: 3493-500, 2014.
5 Huang LW, Lin CY, Lee CC, Liu TZ and Jeng CJ: Overexpression of GRP78 is associated with malignant transformation in epithelial ovarian tumors. Appl Immunohistochem Mol Morphol 20: 381-385, 2012.

6 Zhang J, Jiang Y, Jia Z, Li Q, Gong W and Wang L: Association of elevated GRP78 expression with increased lymph node metastasis and poor prognosis in patients with gastric cancer. Clin Exp Metastasis 23: 401-410, 2006.

7 Kuroda K, Horiguchi A, Asano T, Ito K, Asakuma J, Sato A, Yoshii H, Hayakawa M, Sumitomo M and Asano T: Glucoseregulated protein 78 positivity as a predictor of poor survival in patients with renal cell carcinoma. Urol Int 87: 450-456, 2011.

8 Wu CT, Wang WC, Chen MF, Su HY, Chen WY, Wu CH, Chang YJ and Liu HH: Glucose-regulated protein 78 mediates hormoneindependent prostate cancer progression and metastasis through maspin and COX-2 expression. Tumour Biol 35: 195-204, 2014.

9 Pancewicz-Wojtkiewicz J: Epidermal growth factor receptor and notch signaling in non-small-cell lung cancer. Cancer Medicine 5: 3572-3578, 2016.

10 Pancewicz-Wojtkiewicz J, Eljaszewicz A, Kowalczuk O, Niklinska W, Charkiewicz R, Kozłowski M, Miasko A and Moniuszko M: Prognostic significance of Notch ligands in patients with non-small cell lung cancer. Oncol Lett 13: 506-510, 2017.

11 Cai B, Tomida A, Mikami K, Nagata K and Tsuruo T: Downregulation of epidermal growth factor receptor-signaling pathway by binding of GRP78/BiP to the receptor under glucose-starved stress conditions. J Cell Physiol 177: 282-288, 1998.

12 Endoh H, Tomida S, Yatabe Y, Konishi H, Osada H, Tajima K, Kuwano H, Takahashi T and Mitsudomi T: Prognostic model of pulmonary adenocarcinoma by expression profiling of eight genes as determined by quantitative real-time reverse transcriptase polymerase chain reaction. J Clin Oncol 22: 811-819, 2004.

13 Chao TT, Wang CY, Chen YL, Lai CC, Chang FY, Tsai YT, Chao $\mathrm{CH}$, Shiau CW, Huang YC, Yu CJ and Chen KF: Afatinib induces apoptosis in NSCLC without EGFR mutation through Elk-1mediated suppression of CIP2A. Oncotarget 6: 2164-2179, 2015.

14 Liu X, Lv Z, Zou J, Liu X, Ma J, Wang J, Sa N, Jing P and Xu $\mathrm{W}$ : Afatinib down-regulates MCL-1 expression through the PERK-eIF2 $\alpha$-ATF4 axis and leads to apoptosis in head and neck squamous cell carcinoma. J Cancer Res 6: 1708-1719, 2016.

15 Mao X, Chen Z, Zhao Y, Yu Y, Guan S, Woodfield SE, Vasudevan SA, Tao L, Pang JC, Lu J, Zhang H, Zhang F and Yang J: Novel multi-targeted ErbB family inhibitor afatinib blocks EGF-induced signaling and induces apoptosis in neuroblastoma. Oncotarget 8: 1555-1568, 2017.

16 Dadey DYA, Kapoor V, Hoye K, Khudanyan A, Collins A, Thotala D and Hallahan DE: Antibody targeting GRP78 enhances the efficacy of radiation therapy in human glioblastoma and non-small-cell lung cancer cell lines and tumor models. Clin Cancer Res 23: 2556-2564, 2017.

17 Luo C and Qiu J: MiR-181a inhibits cervical cancer development via downregulating GRP78. Oncol Res, 2017. doi: 10.3727/096504017X14867268787969. [Epub ahead of print]

18 Kawiak A, Domachowska A, Jaworska A and Lojkowska E: Plumbagin sensitizes breast cancer cells to tamoxifen-induced cell death through GRP78 inhibition and Bik upregulation. Sci Rep 13: 43781, 2017.

Received May 11, 2017

Revised May 30, 2017

Accepted May 31, 2017 